"NOTICE: this is the author's version of a work that was accepted for publication in Journal of Biomechanics. Changes resulting from the publishing process, such as peer review, editing, corrections, structural formatting, and other quality control mechanisms may not be reflected in this document. Changes may have been made to this work since it was submitted for publication. A definitive version will be subsequently published in Journal of Biomechanics, [VOL42, pp. 838-84,(2009)]."

\title{
Red blood cell motions in high-hematocrit blood flowing through a stenosed microchannel
}

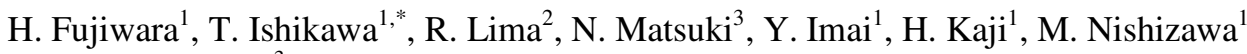 \\ and T. Yamaguchi ${ }^{3}$ \\ ${ }^{1}$ Department of Bioengineering and Robotics, Graduate School of Engineering, Tohoku \\ University, 6-6-01 Aoba, Sendai 980-8579, Japan \\ ${ }^{2}$ Department of Mechanical Technology, ESTiG, Braganca Polytechnic Institute, C. Sta. \\ Apolonia, Braganca 5301-857, Portugal \\ ${ }^{3}$ Department of Biomedical Engineering, Graduate School of Biomedical Engineering, \\ Tohoku University, 6-6-01 Aoba, Sendai 980-8579, Japan
}

\begin{abstract}
We investigated the behaviour of red blood cells (RBCs) in a micro-channel with stenosis by using a confocal micro-PIV system. We could successfully measure individual trajectories of RBCs in a concentrated suspension up to $20 \%$ hematocrit $(\mathrm{Hct})$. The results show that the trajectories of healthy RBCs become asymmetric before and after the stenosis, though trajectories of tracer particles in pure water are almost symmetric. The asymmetry is larger in a $10 \%$ Hct case than in a $20 \%$ Hct case. We also investigated the effect of deformability of RBCs on the trajectories by hardening RBCs by glutarardehyde treatment. The results indicate that the deformability is the key factor in the asymmetry of trajectories and the thickness of cell-free layer. We think that the present results give fundamental knowledge for better understanding blood flow in microcirculations.
\end{abstract}

\section{INTRODUCTION}

Blood flow through a stenosed artery has been investigated widely (Berger \& Jou, 2000; $\mathrm{Ku}, 1997$; Ishikawa et al., 2000, for instance), because the fluid dynamics plays an important role in the progress of arteriosclerosis and infarcts. Most of former hemodynamical studies investigated blood flow in a large artery, although occlusion of small arteries is also important. Lacunar infarcts (Fisher, 1965), for example, are small and deep cerebral infarcts resulting from occlusion of penetrating cerebral arteries, which has the diameter of about $100 \square \mathrm{m}$. Since such a small infarct causes a serious damage to brain, it has been investigated intensively (Wardlaw et al., 2003; Wong et al., 2001; Tanaka et al., 1999; Longstreth et al., 1998; Cacciatore et al., 1991).

In a small artery, say $100 \square \mathrm{m}$ in diamter, the blood is no longer assumed as a homogeneous fluid, because the size of blood cells cannot be neglected compared to the generated flow field. (The diameter of a red blood cell (RBC) is about $8 \square \mathrm{m}$.) In such a case, we need to treat the blood as a multi-phase fluid, and investigate the motion of individual cells in discussing the flow field. Blood may be modelled as a suspension of red blood cells (RBCs) in plasma, because about $99 \%$ of volume fraction of blood cells is RBCs. The interaction of RBCs generates micron-scale mixing in the blood flow, which has a significant effect on diffusion of platelets and large molecules (Ahuja et al., 1978). In our former study, we experimentally 
investigated the self-diffusion of RBCs in a circular glass tube (Lima et al., 2008b), and showed that the diffusion was strongly influenced by the flow field.

In order to measure blood flow experimentally, various methods have been employed, such as double-slit photometric (Gaehtgens et al., 1970; Baker et al., 1974), video microscopy and image analysis (Bugliarello et al. 1963; Goldsmith 1971; Parthasarathi et al., 1999), laserDoppler anemometer (Einav et al., 1975; Born et al., 1978), particle-measuring methods (Sugii et al., 2005; Nakano et al., 2003). These techniques enable us to visualize the flow field, but they are difficult to observe RBCs' behavior inside of the high hematocrit (Hct) blood flow. Because the optical transparency of the RBCs can be very small, even if the flow speed is low. To overcome this problem, we have used a confocal micro-PIV (Particle Image Velocimetry) system (Lima et al., 2006, 2007, 2008a, b). This system enables us to visualize the individual RBCs even in the high Hct blood over $10 \%$ by exciting the labeled RBCs by the laser in the system.

In this study, we investigate the motion of RBCs in a micro-channel with stenosis by using the confocal micro-PIV system. Faivre et al. (2006) investigated similar phenomena, i.e. blood flow through a micro-channel with narrowing, though their research aimed to develop a microfluidic device for separating RBCs and plasma. They measured trajectories of RBCs with the Hct of up to $3 \%$, which is much less than the physiological condition. In this study, we measure individual trajectories of RBCs around the stenosis under high Hct conditions (up to $20 \%$ ), where interactions between RBCs become significant. We discuss the effect of Hct on the flow field and cell-free layers. We also discuss the effect of deformability of RBCs on the trajectories by hardening RBCs by glutarardehyde treatment.

\section{METHODS AND MATERIALS}

\subsection{Confocal micro-PIV system}

The confocal micro-PIV system used in this study (Fig.1) is the same as Lima et al. (2006), so only a brief explanation will be made here. It is consisted of inverted microscope (IX71;Olympus, Tokyo, Japan), confocal scanning system (CSU22;Yokogawa, Tokyo, Japan), high speed camera (Phantom v7.1; Vision Research, NJ, USA), DPSS laser (Laser Quantum, Cheshire, UK), syringe pump (KD Scientific, Holliston, MA, USA) for making constant flow, thermo plate (Tokai Hit, Shizuoka, Japan) for controlling the temperature, and Objective Lens (Olympus, Tokyo, Japan (magnification:20times, N.A.:0.75, W.D.:0.17[mm])). The estimated thickness of the measurement plane (optical slice thickness) of this objective lens is $4.97 \mu \mathrm{m}$. By exposuring the laser to the labeled RBCs, the system enables us to track individual RBCs inside the blood flow of up to $20 \%$ Hct with high resolution and with thin optical thickness.

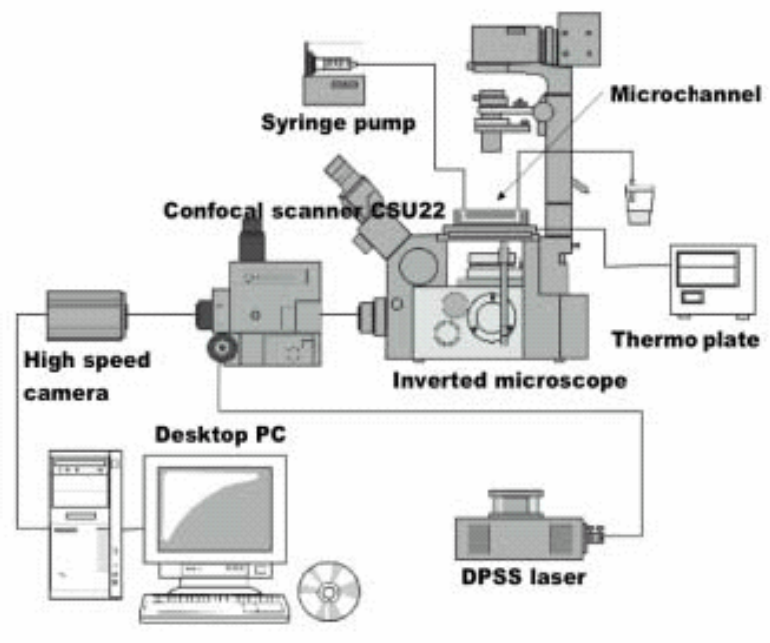

Fig. 1 Schematics of experimental set-up 


\subsection{Materials}

The microchannel is made by polydimethylsiloxane (PDMS) using a soft lithographic technique in the same manner with Lima et al. (2008a). The microchannel has a square cross section with the side length of $50 \mu \mathrm{m}$, and the stenosis has $35 \mu \mathrm{m}$ in height and $30 \mu \mathrm{m}$ in width as

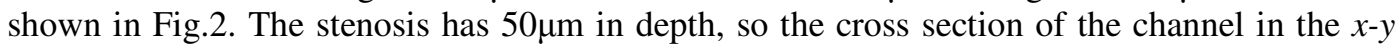
plane does not vary in $z$-direction.

Four kinds of working fluids were used in this study: (a) pure water with $1 \%$ fluorescent particles, (b) dextran 40 (DEX40) with 20\% human RBCs, where 10\% of RBCs are labelled by fluorescent dye (C-7000, Molecular Probes, USA), (c) DEX40 with 10\% human RBCs, where $10 \%$ of RBCs are labelled by fluorescent dye, and (d) DEX40 with $10 \%$ hardened RBCs by glutaraldehyde treatment (Drochon et al., 2003). In the glutaraldehyde treatment, RBCs were suspended at $9 \%$ Hct in Tris buffer containing $0.05 \%$ w/w glutaraldehyde. They were placed in a water bath at 37 degree Celsius during $15 \mathrm{~min}$. The treated RBCs were then centrifuged, washed and re-suspended in the Dextran solution.

The RBCs are taken from a male volunteer, who is 23 years old, and they are centrifuged to separete the RBCs and plasma completely. Then, they are preserved at 4 degree before the start of the experiment dispesed in the pure saline. All procedures in this experiment were carried out in compliance with the Ethics Committee on Clinical Investigation of Tohoku University.

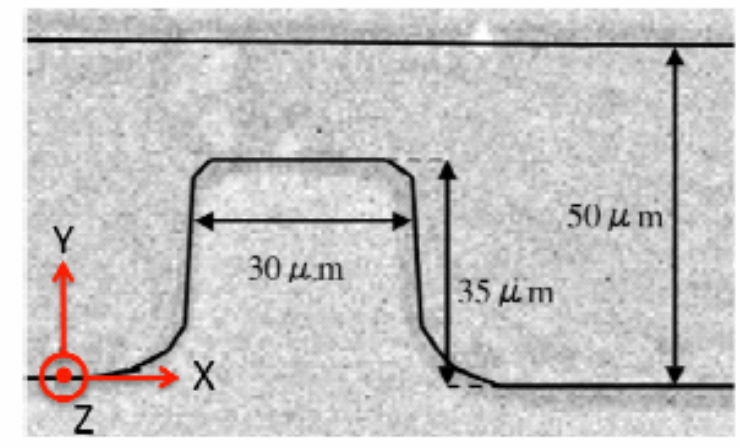

\footnotetext{
Fig.2 : Geometry of the stenosis; the cross section of the channel is a square with the side length of $50 \mu \mathrm{m}$, and the stenosis has $35 \mu \mathrm{m}$ in height, $30 \mu \mathrm{m}$ in width and $50 \mu \mathrm{m}$ in depth.

The $x, y, z$-coordinates are taken as shown in the figure. Shape of the stenosis does not vary in the $z$-direction.
}

\subsection{Experimantal conditions}

Throughout this study, the average velocity is kept at about $0.45 \mathrm{~mm} / \mathrm{s}$. Reynolds number based on the average velocity and the side length of the square cross section is about 0.2 , which is a typical value of in vivo condition for small arteies with $50 \mu \mathrm{m}$ diameter (cf. Popel and Johnson, 2005). The micro-channel is surround by adiabatic walls, and the temperature of the channel is maintained at 37 degree Celsius by the thermo plate. The frame rate for the high speed camera is 300 frame per second. We observed the motion of RBCs in the center plane, i.e. $25 \mu \mathrm{m}$ from the bottom.

\section{RESULTS}

\subsection{Flow of pure water}

First, we investigated the flow field of pure water. Fluorescent particles are added to the pure water, and they are visualized by the confocal micro-PIV system as shown in Fig.3(a). The large arrow in the figure indicates the flow direction. The trajectories of tracer particles can be measured by continuously tracking individual particles as shown in Fig.3(b). We see that the 
trajectories are almost symmetric between the upstream and the downstream of the stenosis. This result is consistent with the Stokes flow condition, where the streamlines become symmetric if the stenosis shape is symmetric to the $y$-axis.

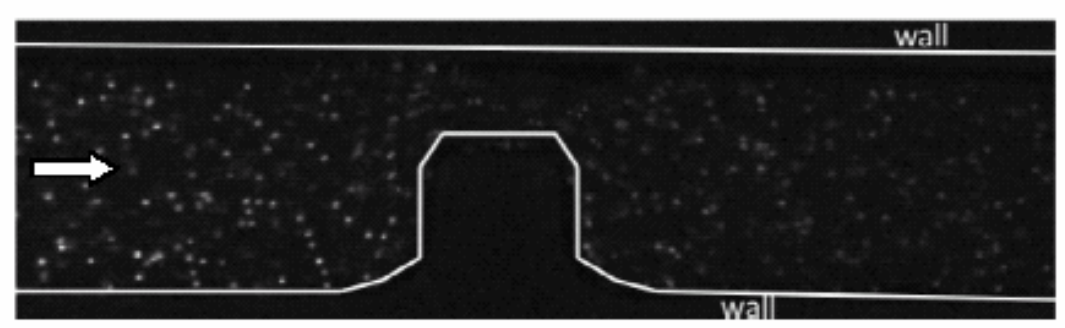

(a) A sample of original confocal image

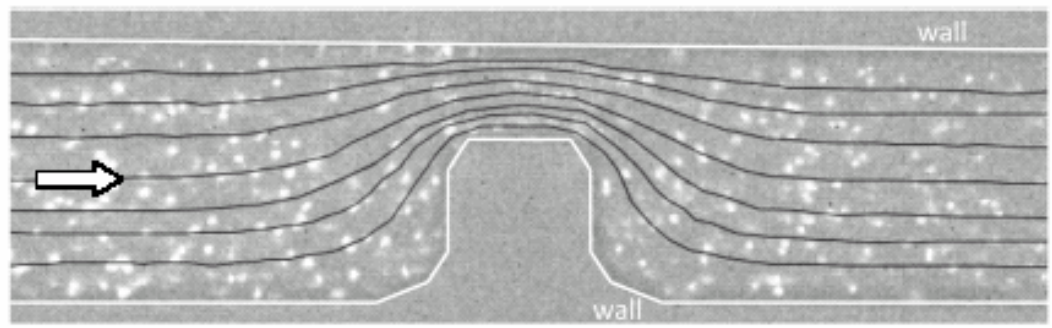

(b) Trajectories of tracer particles

Fig. 3 : Flow of pure water with $1 \%$ fluorescent particles. The lange arrows in the figure indicate the flow direction

\subsection{Flow of $10 \%$ Hct blood with healthy RBCs}

Next, we investigated the blood flow. Labelled RBCs in the blood flow with $10 \%$ Hct were visualized at the centre plane, i.e. $25 \mu \mathrm{m}$ depth in the $z$-direction, and a sample of original confocal image is shown in Fig.4(a). We see that the labelled RBCs are clearly visualized. The trajectories of individual RBCs can be measured by continuously tracking them, and the results are shown in Fig.4(b). We see that the trajectories of RBCs are no longer symmetric. RBCs initially locating near the lower wall tend to move away from the wall after the stenosis. Such position change in the $y$-direction is induced by the interaction between the RBC and the stenosis wall. RBCs initially locating near the upper wall, on the other hand, again tend to move away from the wall after the stenosis. This position change in the $y$-direction is induced by the interaction between the RBC and the upper wall. It is found that the $y$ position of RBCs change considerably in a blood flow through the stenosed micro-channel.

The asymmetry of the trajectories are induced by the deformation of RBCs (this will be confirmed by comparing with the results of hardened RBCs in section 3.4 below). RBCs are deformed by the traction forces generated by the inner- and outer-fluid of the membrane, which is dependent on the history of deformation along the trajectories. Thus, the deformation of a $\mathrm{RBC}$ becomes asymmetric before and after the stenosis, even though the flow field is governed by the Stokes equation. This is the reason why trajectories of RBCs show asymmetry before and after the stenosis. 


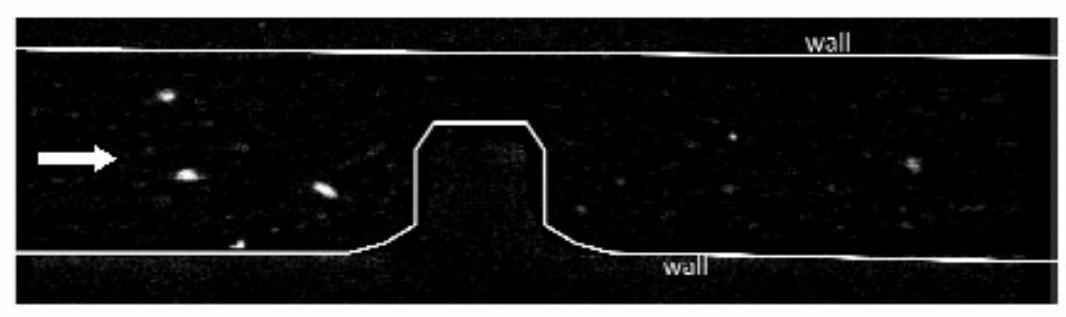

(a) A sample of original confocal image

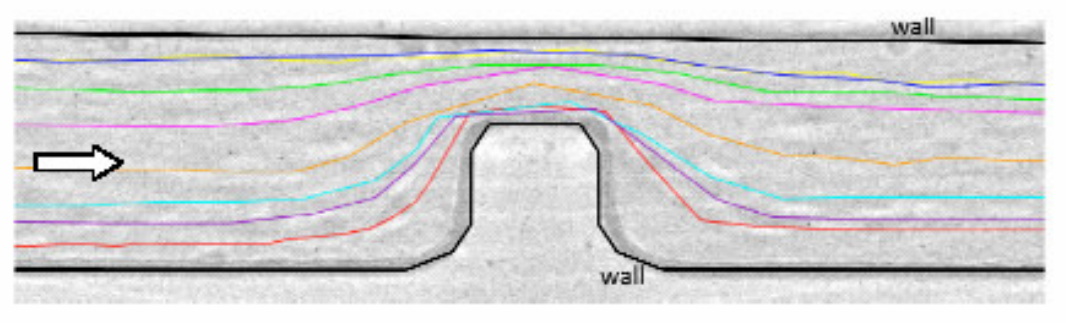

(b) Trajectories of labeled RBCs

Fig. 4 : Flow of blood with $10 \%$ Het. The lange anrows in the figure indicate the flow direction

\subsection{Flow of $20 \%$ Hct blood with healthy RBCs}

We increased Hct up to $20 \%$, and measured the trajectories labelled RBCs in a blood flow. The results are shown in Fig.5. We see that the trajectories are not as asymmetric as those of Hct $=10 \%$. In order to discuss this tendency quantitatively, we measured the RBC displacement in the $y$-direction induced by the flow through stenosis. We define $\square \mathrm{Y}$ as $\square \mathrm{Y}=\mathrm{Y}_{\text {upstream }}-$ $\mathrm{Y}_{\text {downstream}}$, where $\mathrm{Y}_{\text {upstream }}$ and $\mathrm{Y}_{\text {downstream }}$ are the $y$ position of a labelled RBC $60 \square \mathrm{m}$ upstream and downstream of the stenosis, respectively (see Fig.6). The results of 10\% Hct and 20\% Hct with various $Y_{\text {upstream }}$ are shown in Fig.7. The error bars indicate standard deviations. In the case of $10 \% \mathrm{Hct}, \mathrm{RBCs}$ near the lower wall, i.e. $\mathrm{Y}_{\text {upstream }}$ of about $0-10$, show significant displacement away from the wall. RBCs near the upper wall, i.e. $Y_{\text {upstream }}$ of about 40-50, also show displacement away from the wall (this time, the sign of $\square \mathrm{Y}$ is negative). These results indicate that the RBCs tend to flow in the core region of the bulk flow after flowing through the stenosis, though RBCs initially located in the core region do not move in the $y$-direction significantly. In the case of $20 \% \mathrm{Hct}$, however, the absolute value of $\square \mathrm{Y}$ is much smaller than that of $10 \%$ Hct case. When Hct is high, the interactions between RBCs increase considerably. Thus, the RBCs cannot concentrate in the core region, but pushed away from the high concentrated region due to the interaction. As a result, the displacement of RBCs may be suppressed more in the $20 \%$ Hct case. Of course, the dispersion of RBCs due to interaction is increased as Hct is increased, as discussed in Lima et al. (2008b). But the dispersion is isotropic and does not help RBCs to move in one direction. 


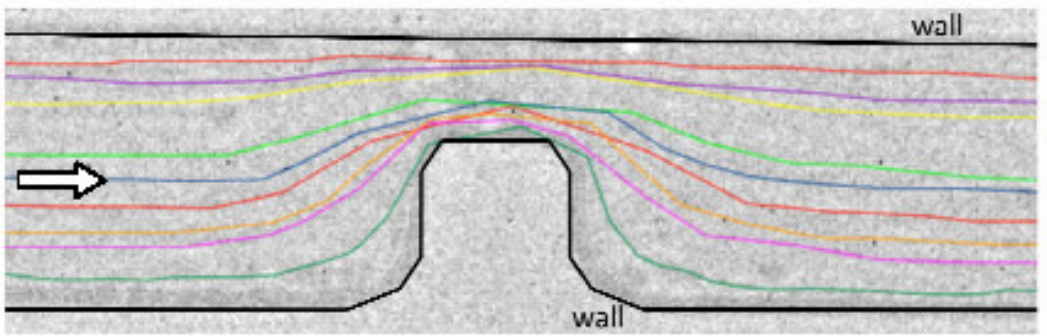

Fig.5: Trajectories of labeled RBCs in a blood flow with $20 \%$ Het. The large arrow in the figure indicates the flow direction

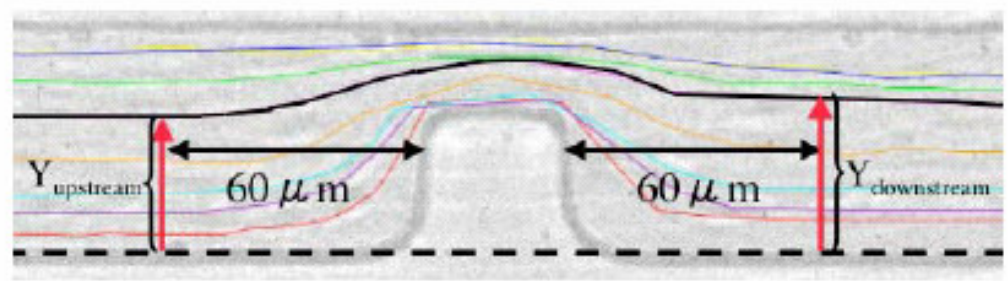

Fig.6 : Definition of $Y_{\text {upsineam }}$ and $Y_{\text {dowistream }}$

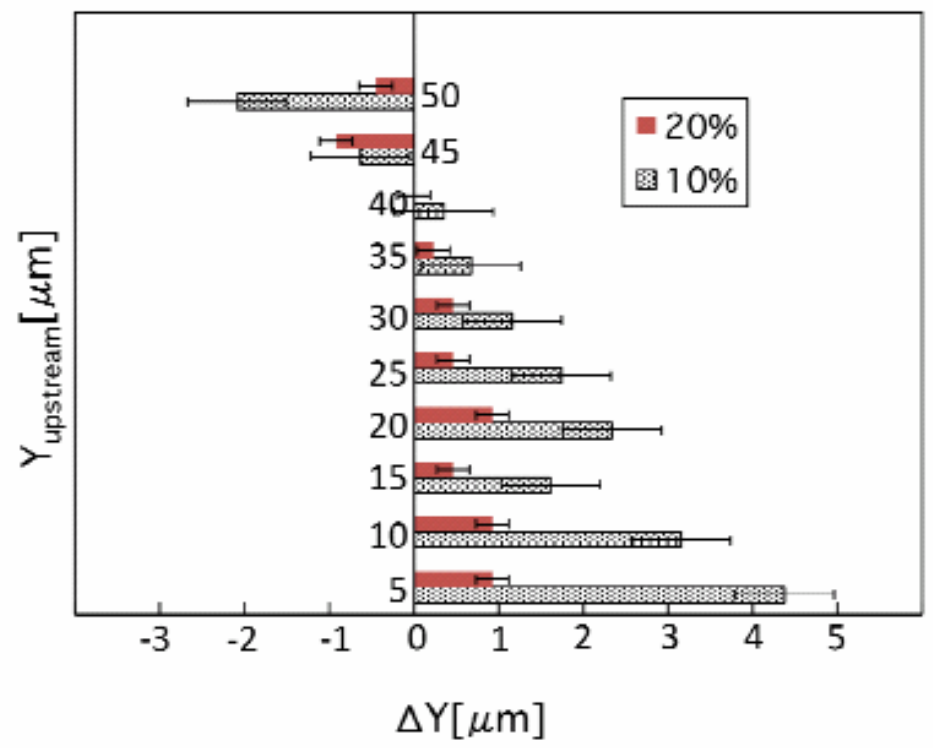

Fig.7 Effect of Hct and $Y_{\text {upstrean }}$ on $\Delta Y$. The error bars indicate standard deviations.

\subsection{Flow of $10 \%$ Hct blood with hardened RBCs}

Lastly, we investigated the blood flow with hardened RBCs in order to clarify the effect of cell deformability on the blood flow. The RBCs are hardened by glutaraldehyde treatment as explained in section 2.2. Since the hardened RBCs are not labelled by fluorescent dye, we could not track them under the condition of $10 \%$ Hct. Instead, we measure the cell-free layer on the upper and lower wall downstream of the stenosis. The RBCs near the wall can be visualized by 
using normal halogen light, because the concentration of RBCs can be low near the wall and the image of RBCs do not overlap so often. We measure the distance between upper or lower wall and the nearest RBC from the wall at 50,80 and $110 \square \mathrm{m}$ downstream of the stenosis. By taking average of the distance for about 60 RBCs per wall, we calculate thickness of the cell-free layer. The results are shown in Fig. 8 with the error bar of the standard deviation. It is found that the hardened RBCs show considerably thinner cell-free layer compared to the healthy RBCs. If RBCs are hardened, the asymmetry in trajectories is no longer enhanced by the deformation of RBCs. Thus, hardened RBCs initially flowing near the wall can stay near the wall even after flowing through the stenosis. We can conclude, therefore, that the deformability of RBCs plays an important role in the blood flow through the stenosis.

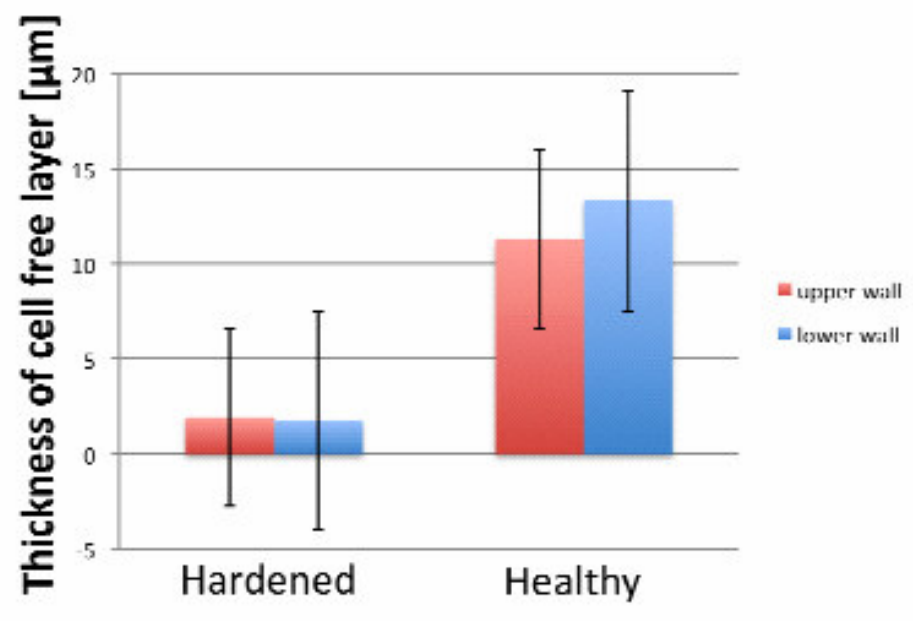

Fig.8 Effect of the deformability of RBCs on the cell-free layer near the upper and lower wall downstream of the stenosis $(\mathrm{Het}=10 \%)$. The error bars indicate standard deviations.

\section{DISCUSSIONS}

The present results indicate that the deformability of RBCs enhance the displacement perpendicular to the wall when they flow through a stenosis. This displacement is induced by the interaction between RBCs and the stenosed wall. Though it is a different problem, the similar tendency has been recognized widely for the interaction of two particles in a shear flow. If one puts two rigid spheres in a simple shear flow, they interact hydrodynamically. The trajectories of two spheres, however, are symmetric before and after the interaction, as derived by Batchelor and Green (1972). In contrast, if the particles are deformable, such as droplets and capsules, the trajectories become asymmetric. The displacement perpendicular to the flow, before and after the interaction, has been investigated numerically (Lac et al., 2007, for instance). The results showed that the deformability was the key factor for the asymmetry in the trajectories. The mechanism of the asymmetry in trajectories can be explained by the asymmetry in the deformation before and after the near-contact. In the present study, the trajectories of healthy RBCs are much more asymmetric than those of hardened RBCs. Our results support those former studies, though the problem discussed here is the interactions between a deformable particle and a solid wall, instead of two deformable particles.

As mentioned in Introduction, Faivre et al. (2006) also investigated blood flow through a micro-channel with narrowing in order to develop a microfluidic device for separating RBCs and plasma. In their experiment, they observed that the thickness of cell-free layer increases as Hct is increased. This tendency is the opposite from our results. We think that the difference in tendency may be caused by the difference in hematocrit used in two studies; Faivre et al. used a dilute blood $(\mathrm{Hct}=3 \%)$, whereas we used more concentrated blood $(\mathrm{Hct}=10,20 \%)$. In a high Hct blood, the hydrodynamic interactions between RBCs are important, and the behavior of individual RBCs becomes different from that in a low Hct blood. We think that RBCs tends to 
be pushed away from the concentrated core region when Hct is high, which tends to reduce the thickness of the cell-free layer.

Even in microcirculations in vivo, Peclet number for mass transport of large molecules and platelets becomes larger than unity (Ahuja et al., 1978). Thus, the mass transport is not only governed by the Brownian diffusion but also by the micron-scale flow field generated by interactions of RBCs. The present results suggest that RBCs tend to flow away from the wall after the stenosis, which may affect mass transport of large molecules and platelets around the stenosis. Since the mixing near the wall is enhanced by the micron-scale flow field generated by RBCs, the cell-free layer may suppress mass transport. This means that the effective diffusion coefficient for large molecules and platelets may vary spatially around the stenosis. This kind of local change in mass transport condition in microcirculation has not been clarified so far. We think that the present results give fundamental knowledge for better understanding blood flow and mass transport in microcirculations.

\section{CONCLUSIONS}

In this study, we investigated the behaviour of RBCs in a micro-channel with stenosis by using the confocal micro-PIV system. We could successfully measure individual trajectories of RBCs around the stenosis up to $20 \%$ Hct. The results show that the trajectories of healthy RBCs become asymmetric before and after the stenosis, though trajectories of tracer particles in pure water are almost symmetric. The asymmetry is larger in the $10 \%$ Hct case than in the $20 \% \mathrm{Hct}$ case. We also investigated the effect of deformability of RBCs on the trajectories by hardening RBCs by glutaraldehyde treatment. The results indicate that the deformability is the key factor in the asymmetry of the trajectories and the thickness of the cell-free layer. We think that the present results give fundamental knowledge for better understanding blood flow in microcirculations.

\section{ACKNOWLEDGMENTS}

This research was supported by Grants in Aid for Scientific Research(S) from JSPS No. 19100008, and by Grant-in-Aid for Young Scientists (A) from JSPS No.19686016. We also acknowledge the support of 2007 Global COE Program "Global Nano-Biomedical Engineering Education and Research Network Centre", and "the Development and Use of the NextGeneration Supercomputer Project" from the Ministry of Education, Culture, Sports, Science and Technology (MEXT).

\section{REFERENCES}

Ahuja, A. S., Hendee, W. R. and Carson, P. L., Transport phenomena in laminar flow of blood, Phys. Med. Biol., 23, pp.928-936 (1978)

Baker M. and Wayland H., On-line volume flow rate and velocity profile measurement for blood in microvessels, Microvasucular Res.. 7, pp.131-143 (1974)

Batchelor, G. K. and Green, J. T., The hydrodynamic interaction of two small freely-moving spheres in a linear flow field, J. Fluid Mech., 56, pp.375-400 (1972).

Berger, S. A. and Jou, L-D, Flows in stenotic vessels, Annu. Rev. Fluid Mech., 32, pp.347-382 (2000)

Born G., Melling A. and Whitelaw J., Laser Doppler microscope for blood velocity measurement, Biorheology. 15, pp.163-172 (1978)

Bugliarello G. and Hayden J., Detailed characteristics of the flow of blood in vitro, Trans. Soc. Rheol. 7, pp.209-230 (1963) 
Cacciatore, A. and Russo L. S. Jr, Lacunar Infarction as an Embolic Complication of Cardiac and Arch Angiography, Stroke, 22, pp.1603-1605 (1991)

Drochona A., Rheology of dilute suspensions of red blood cells: experimental and theoretical approaches, Eur. Phys. J. AP, 22, pp.155-162 (2003)

Einav S., Berman R., Fuhro P., DiGiovannni P., Fine S. and Fridman J., Measurement of velocity profiles of red blood cells in the microcircualation by laser Doppler anemometry (LDA), Biorheology. 12, pp.207-210 (1975)

Faivre, M., Abkarian, M., Bickraj, K., Stone, H. A., Geometrical focusing of cells in a microfluidic device: An approach to separate blood plasma, Biorheology, 43, pp.147-159 (2006)

Fisher, C. M., Lacunes: small, deep cerebral infarcts, Neurology, 15, pp.774-784 (1965).

Gaehtgens P., Meiselman H. and Wayland H., Velocity profiles of human blood at normal and reduced hematocritin glass tubes up to $130 \mu \mathrm{m}$ diameter, Microvasucular Res.. 2, pp.13-23 (1970)

Goldsmith H., Red cell motions and wall interactions in tube flow, Federation Proc.. 30, pp.1578-1588 (1971)

Ishikawa, T., Oshima, S. and Yamane, R., Vortex enhancement in blood flow through stenosed and locally expanded tubes, Fluid Dyn. Res., 26, pp.35-52, (2000)

Ku, D. N., Blood flow in arteries, Annu. Rev. Fluid Mech., 29, pp.399-434 (1997)

Lac, E., Morel, A. and Barthes-Biesel, D., Hydrodynamic interaction between two identical capsules in simple shear flow, J. Fluid Mech., 573 , pp.149-169 (2007)

Lima, R., Wada, S., Tsubota, K. and Yamaguchi, T., Confocal micro-PIV measurements of three-dimensional profiles of cell suspension flow in a square microchannel, Meas. Sci. Tecnol. 17, pp.797-808 (2006)

Lima, R., Wada, S., Takeda, M., Tsubota K. and Yamaguchi, T., In vitro confocal micro-PIV measurements of blood flow in a square microchannel: The effect of the hematocrit on instantaneous velocity profiles, J. Biomech., 40, pp.2752-2757 (2007)

Lima, R., Wada, S., Tanaka, S., Takeda, M., Ishikawa, T., Tsubota, K., Imai K. and Yamaguchi, T., In vitro blood flow in a rectangular PDMS microchannel: experimental observations using a confocal micro-PIV system, Biomed. Microdev., 10, pp.153-167 (2008a)

Lima, R., Ishikawa, T., Imai, Y., Takeda, M., Wada, S. and Yamaguchi, T., Radial dispersion of red blood cells in blood flowing through glass capillaries: Role of Heamatocrit and geometry, $J$. Biomech., 41, pp.2188-2196 (2008b)

Longstreth, W. T. Jr, Bernick, C., Manolio, T. A., Bryan, N., Jungreis, C. A. and Price, T. R., Lacunar Infarcts Defined by Magnetic Resonance Imaging of 3660 Elderly People, The Cardiovascular Health Study, Arch Neurol., 55, pp.1217-1225 (1998)

Nakano A., Sugii Y., MInamiyama M. and Niimi H., Measurement of red cell velocity in microvessels using particle image velocimetry(PIV), Clinical Hemorheol. Microcircul.. 29, pp.445-455 (2003) 
Parthasarathi A., Japee S. and Pittman R., Determination of red blood cell velocity by video shuttering and image analysis. Anna. Biomed. Eng.. 27, pp.313-325 (1999)

Popel, A. S. and Johnson, P. C., Microcirculation and hemorheology, Ann. Rev. Fluid Mech., 37, pp.43-69 (2005)

Sugii Y., Okuda R., Okamoto K. and Madarame H., Velocity measurement of both red blood cells and plasma of in vitro blood flow using high-speed micro PIV technique, Measurement Sci. Tech.. 16, pp.1126-1130 (2005)

Tanaka, E., Tanaka, A., Sekka, T., Shinozaki, Y., Hyodo, K., Umetani, K. and Mori, H., Digitized Cerebral Synchrotron Radiation Angiography: Quantitative Evaluation of the Canine Circle of Willis and Its Large and Small Branches, AJNR Am J Neuroradiol, 20, pp.801-806 (1999)

Wardlaw, J. M., Sandercock, P. A. G., Dennis, M. S. and Starr, J., Is Breakdown of the BloodBrain Barrier Responsible for Lacunar Stroke, Leukoaraiosis, and Dementia?, Stroke, 34, pp.806-812 (2003)

Wong, E. H., Pullicino, P. M. and Benedict, R., Deep Cerebral Infarcts Extending to the Subinsular Region, Stroke, 32, pp.2272-2277 (2001) 